\title{
La traduction en droit positif : les régimes linguistiques en droit communautaire
}

\author{
Anne-Lise SiBONY* et Nicolas DE SADELEER ${ }^{* *}$
}

\section{Introduction}

En droit communautaire, la traduction, avant de se présenter comme une métaphore ou un paradigme ${ }^{1}$, est une obligation de droit positif $^{2}$. La présente contribution vise à en préciser les contours à travers l'étude du régime linguistique applicable aux institutions, mais aussi des règles de droit matériel dont découlent pour les opérateurs économiques et pour les justiciables des obligations de traduction, qu'il s'agisse de faire traduire l'étiquette apposée sur un produit, un contrat de travail ou encore une assignation en justice.

L'étude des obligations de traduction imposées par le droit communautaire présente une difficulté principale, liée à la dispersion des sources de ces obligations à travers le droit primaire, le droit dérivé et les principes généraux du droit. Très peu de dispositions de droit primaire sont consacrées au régime linguistique des Communautés. En outre, les dispositions générales de droit dérivé, également rares, ne

Chargée de cours, ULg.

** Professeur, FUSL, professeur invité à l'UCL et à l'université Paris II.

1 Sur la traduction comme paradigme scientifique au sens de Kuhn, Fr. OsT, Traduire: Défense et illustration du multilinguisme, Fayard, Paris, coll. "Ouvertures », 2009, p. 11-12.

2 La traduction est bien sûr aussi un défi pour l’Union européenne. Voy. J. RIDEAU, «L'Union européenne face aux défis linguistiques», in J.-J. SUEUR (dir.), Interpréter et traduire. Actes du colloque international des 25 et 26 novembre 2005, faculté de droit de Toulon, Bruylant, Bruxelles, 2007, p. 63-138. 
traitent que des relations entre institutions et particuliers, si bien qu'un grand nombre de situations sont régies par des textes spéciaux ou par des solutions jurisprudentielles élaborées à partir de principes généraux du droit. Ceux-ci sont multiples et, partant, susceptibles de conduire à des orientations parfois divergentes. Ainsi, le principe de non-discrimination, qui tend à étendre les obligations de traduction, doit être concilié avec des préoccupations économiques, qui tendent à l'inverse à restreindre ces obligations.

L'objet du présent chapitre est de donner un aperçu des obligations de traduction imposées par le droit communautaire positif, à la fois dans son volet institutionnel et dans son volet matériel. Pour cela, il convient de présenter tout d'abord les régimes linguistiques des institutions (I), puis la manière dont les exigences de traduction se combinent avec les libertés économiques garanties par les traités (II). Enfin, quelques exemples illustreront quelles questions de traduction se posent dans un domaine encore en développement au sein du droit communautaire, celui de la coopération judiciaire en matière civile (III).

\section{Le régime linguistique des institutions}

Force est de constater que le régime linguistique des institutions n'est pas fixé comme tel dans le traité $\mathrm{CE}$, lequel habilite en revanche le Conseil, statuant à l'unanimité, à définir ce régime (art. $290 \mathrm{CE}$, art. 342 TFUE). Par conséquent, le régime linguistique des institutions relève principalement du droit dérivé et plus spécialement du «règlement numéro 1 », qui en forme le socle (A). En effet, le choix des langues dans lesquelles les institutions travaillent et s'expriment étant une question préalable à leur prise de fonction, c'est le tout premier règlement communautaire qui fut consacré à cette question ${ }^{3}$. Ce règlement ne réglait toutefois pas toutes les situations et la jurispru-

\footnotetext{
Règlement $\mathrm{n}^{\circ} 1$ portant fixation du régime linguistique de la Communauté économique européenne, JO L 17 du 6 octobre1958, p. 385-386. Dernière version consolidée (2007) disponible sur le site EUR-Lex: http://eur-lex.europa.eu/ LexUriServ/LexUriServ.do?uri=CONSLEG:1958R0001:20070101:FR:PDF (consulté en dernier lieu le 20 juillet 2009).
} 
dence l'a complété à partir de principes généraux du droit (B). Si le règlement $\mathrm{n}^{\circ} 1$ et les principes généraux du droit sont les principales sources du régime linguistique des institutions, il convient toutefois de mentionner d'autres dispositions plus ponctuelles et assez éparses, puisqu'elles sont issues tant du traité que du droit dérivé et de la Charte des droits fondamentaux (C).

\section{A. Le socle du régime juridique des institutions : le règlement $n^{\circ} 1$}

L'article 290 CE prévoit que «le régime linguistique des institutions de la Communauté est fixé, sans préjudice des dispositions prévues par le statut de la Cour de justice, par le Conseil statuant à l'unanimité ». C'est sur la base de cette disposition que le règlement $\mathrm{n}^{\circ} 1 \mathrm{du}$ Conseil sur la fixation du régime linguistique de la $\mathrm{CEE}^{4}$, qui détermine les langues officielles et les langues de travail des institutions de la Communauté, a pu être adopté.

Le règlement $\mathrm{n}^{\circ} 1$ fixe les langues officielles de la Communauté, lesquelles sont aujourd'hui au nombre de vingt-trois ${ }^{5}$. Selon l'article 2 de ce texte, le choix par l'administré d'une de ces langues officielles oblige l'institution à lui faire parvenir sa réponse dans la langue qu'il a choisie. En vertu de l'article 3, les écrits adressés à un État membre ou à une personne relevant de la juridiction de cet État doivent être rédigés dans la langue officielle de cet État. Toutefois, la violation de cette exigence n'entache d'irrégularité la procédure au cours de laquelle cet écrit est communiqué qu'à certaines conditions ${ }^{6}$. Les règlements et les autres textes de portée générale sont rédigés dans toutes ces langues (article 4) et le Journal officiel paraît dans toutes les langues officielles (article 5). Ces principes posés dans le règlement $\mathrm{n}^{\circ} 1$ sont restés inchangés depuis l'origine. Seul le nombre de langues officielles a

4 Règlement $n^{\circ} 1$, cité supra note 3.

5 Le bulgare, l'espagnol, le tchèque, le danois, l'allemand, l'estonien, le grec, l'anglais, le français, l'irlandais, l'italien, le letton, le lituanien, le hongrois, le maltais, le néerlandais, le polonais, le portugais, le roumain, le slovaque, le slovène, le finnois et le suédois.

6 La jurisprudence de la Cour sur ce point est présentée infra. 
augmenté à mesure des élargissements successifs. Il en résulte actuellement une importante activité de traduction au sein des institutions ${ }^{7}$.

Le règlement $\mathrm{n}^{\circ} 1$ n'affirme nulle part que toutes les langues de la Communauté doivent être employées dans toutes les situations comme langues de travail de toutes les institutions ${ }^{8}$. Son article 6 permet, en effet, aux institutions de déterminer les modalités d'application du régime linguistique dans leurs règlements intérieurs respectifs ${ }^{9}$. Aussi les règles linguistiques varient-elles d'une institution à l'autre.

Conformément à cet article 6 , le règlement intérieur du Parlement européen prévoit que tous les documents doivent être rédigés dans les langues officielles ${ }^{10}$. Le Conseil ne délibère et ne décide que sur la base de documents rédigés dans toutes les langues officielles, sauf décision contraire prise à l'unanimité et motivée par l'urgence ${ }^{11}$. Même si dans la pratique la Commission applique un régime trilingue (anglais, allemand et français), le règlement intérieur de la Commission prévoit que ses décisions sont arrêtées dans la ou les versions linguistiques qui sont obligatoires en vertu des règles générales pré-

7 On compte actuellement 506 combinaisons linguistiques possibles (23 langues officielles traduisibles dans les 22 autres). A titre d'exemple, la DG Traduction au sein de la Commission emploie à plein temps 1750 traducteurs (dont les activités ne se limite pas à la traduction de textes législatifs). Source: Commission européenne, Traduction et multilinguisme, Office des publications, 2007, disponible sur le site de la DG Traduction: http://ec.europa.eu/dgs/translation/index_fr.htm (consulté en dernier lieu le 20 juillet 2009). Le service de traduction du Parlement européen compte près de 700 traducteurs. Source: site du Parlement européen (consulté en dernier lieu le 20 juillet 2009). De même, le service linguistique du secrétariat général du Conseil compte 700 traducteurs environ. Source : site du Conseil, consulté en dernier lieu le 20 juillet 2009).

8 Conclusions JACOBS sous CJCE, 9 septembre 2003, Kik, C-161/03 P, Rec., p. I8283, point 46 .

9 Ces règlements ne sont pas tous publiés au Journal officiel, mais sont disponibles à partir de la page suivante: http://europa.eu/legislation_summaries/institutional_ affairs/institutions_bodies_and_agencies/index_fr.htm (consulté en dernier lieu le 20 juillet 2009).

10 Article 147 du règlement intérieur du Parlement européen, JO L 44 du 15 février 2005.

11 Article 14, paragraphe 1, du règlement intérieur du Conseil (décision 2004/338/CE, Euratom du Conseil du 22 mars 2004), JO L 106 du 15 avril 2004. 
vues par le règlement $n^{\circ} 1^{12}$. Les documents de la Cour des comptes doivent être rédigés dans les langues officielles ${ }^{13}$. Quant au régime linguistique de la Cour de justice, l'article 290 CE prévoit qu'il est réglé dans son statut. Toutefois, le régime linguistique définitif n'ayant jamais été adopté, l'article 64 du statut de la Cour renvoie sur ce point aux règlements de procédure des différentes juridictions qui la composent (Cour de justice, Tribunal de première instance et Tribunal de la fonction publique) ${ }^{14}$. Ceux-ci disposent que toutes les langues officielles de l'Union peuvent être des langues de procédure et règlent le choix de cette langue par les parties ${ }^{15}$.

Le règlement $\mathrm{n}^{\circ} 1$ n'empêche pas le Conseil de prévoir des régimes juridiques divergents en ce qui concerne les agences de l'Union européenne. Ainsi, en établissant l'Office de l'harmonisation dans le marché intérieur (OHMI), responsable de l'enregistrement des marques et dessins ou modèles, le Conseil a décidé que les demandes de marque communautaire seraient déposées dans une des langues de l'Office, à savoir l'allemand, l'anglais, l'espagnol, le français et l'italien ${ }^{16}$.

12 Article 17 du règlement intérieur de la Commission, version consolidée non publiée au $J O$, disponible à l'url suivante: http://europa.eu/legislation_summaries/ institutional_affairs/institutions_bodies_and_agencies/010004_fr.htm (consultée en dernier lieu le 20 juillet 2009)

13 Article 24, paragraphe 1, du règlement intérieur de la Cour des comptes, JO L 18 du 20 janvier 2005, p. 1.

14 L'article 64 du statut de la Cour dispose que «[j]usqu'à l'adoption de règles relatives au régime linguistique applicable à la Cour et au Tribunal dans le présent statut, les dispositions du règlement de procédure de la Cour et du règlement de procédure du Tribunal relatives au régime linguistique demeurent applicables. Toute modification ou abrogation de ces dispositions doit être faite selon la procédure prévue pour la modification du présent statut. »

15 Articles 29 à 31 du règlement de procédure de la Cour; articles 32 à 37 du règlement de procédure du Tribunal. L'article 29 du règlement de procédure du Tribunal de la fonction publique rend applicable devant cette juridiction les dispositions pertinentes du règlement de procédure du Tribunal de première instance. $J O$ L 225 du 29 août 2007, p. 1. Des versions consolidées des règlements de procédure de la Cour et du Tribunal sont disponibles sur le site de la Cour, respectivement: http://curia.europa.eu/jcms/jcms/Jo2_7031/ et http://curia.europa.eu/jcms/jcms/Jo2_7040/ (consultés en dernier lieu le 20 juillet 2009).

16 Article 115, paragraphe 2 du règlement (CE) n 40/94 du Conseil, du 20 décembre 1993, sur la marque communautaire, JO 1994, L 11, p. 1. 
L'article 3 du règlement $\mathrm{n}^{\circ} 1$ a par ailleurs donné de longue date lieu à une jurisprudence concernant la portée de l'obligation de traduction des documents communiqués par les institutions à un État membre ou à une personne relevant de la juridiction d'un État membre. Cette jurisprudence est dès l'origine empreinte de pragmatisme et limite les cas de nullité pour défaut de traduction. Ainsi, en ce qui concerne les actes des institutions notifiés à un particulier, la Cour a jugé, sans surprise, que leur légalité n'est pas affectée lorsque cet acte est notifié dans la langue de l'État dont relève le destinataire et est en outre accompagné d'une traduction dans une autre langue ${ }^{17}$. De même, en ce qui concerne un écrit transmis à une autorité nationale, la Cour a-t-elle jugé que la procédure au cours de laquelle un écrit non accompagné de la traduction exigée par l'article 3 n'est entachée d'irrégularité que si des conséquences préjudiciables en résultent pour le destinataire de cet écrit, par exemple parce que celui-ci a été empêché d'en prendre utilement connaissance et, par conséquent, de faire valoir son avis dans le cadre de la procédure en cause ${ }^{18}$. En ce qui concerne les procédures d'élaboration des actes communautaires, il arrive que, pour gérer efficacement les réunions des différents comités qui épaulent la Commission, cette dernière transmette les convocations aux nombreuses réunions de ces comités dans un nombre limité de langues officielles. L'efficacité l'emporte alors sur le respect de $l^{\prime}$ article 3 du règlement $n^{\circ} 1$. Dans une hypothèse de ce type, la Cour de justice a jugé que l'absence d'une version allemande de deux annexes à une convocation à la réunion du comité de réglementation, bien qu'elle ne soit pas conforme à cet article, n'entraîne pas pour autant nécessairement l'annulation du règlement attaqué. L'annulation de l'acte finalement adopté n'est encourue que si, en raison de l'absence de la traduction litigieuse, la procédure aurait pu aboutir à un résultat différent ${ }^{19}$.

17 CJCE, 16 décembre 1975, Suiker Unie c. Commission, 40 à 48, 50, 54 à 56, 111, 113 et 114/73, Rec., p. 1663, points 114-115.

18 CJCE, 15 juillet 1970, ACF Chemiefarma c. Commission, 41/69, Rec., p. 661, points 47 à 52 .

19 CJCE, 25 octobre 2005, Allemagne et Danemark c. Commission, C-465/02 et C-466/02, Rec., p. I-9115, points 36 et 37. 
Le Tribunal de première instance a suivi ce raisonnement en jugeant que le non-respect par la Commission de l'article 3 du règlement $\mathrm{n}^{\circ} 1$ n'est pas nécessairement constitutif d'une violation des formes substantielles et n'entache pas nécessairement la validité de l'acte adopté à l'issue d'une procédure de comitologie ${ }^{20}$. En l'espèce, le royaume d'Espagne arguait devant le Tribunal que, en violation de l'article 3 du règlement $\mathrm{n}^{\circ} 1$, la convocation à une réunion d'un comité de gestion ainsi qu'un amendement de fond au texte initial qui lui avaient été adressées par la Commission auraient dû être rédigés en langue espagnole et non pas en anglais. Selon l'État requérant, l'acte adopté par la Commission était entaché de nullité, au motif que des modifications substantielles auraient été apportées au projet au cours de cette réunion, et que ces modifications n'avaient pas été présentées en langue espagnole. Bien que la méconnaissance du régime linguistique découlant du règlement $\mathrm{n}^{\circ} 1$ fut constitutive d'un vice de procédure, le Tribunal a jugé que les convocations adressées par la Commission, en langues anglaise, française et allemande, n'avaient nullement empêché les délégations nationales de participer à la réunion du comité de gestion. Il en a déduit que la procédure d'adoption de l'acte contesté n'aurait pas pu aboutir à un résultat différent en l'absence de l'irrégularité dénoncée. A cette occasion, le Tribunal a également apporté une précision sur le champ d'application de l'article 3 du règlement $\mathrm{n}^{\circ} 1$, jugeant que ce texte n'est pas d'application aux amendements, même de fond, proposés par la Commission au comité de gestion ${ }^{21}$.

$\mathrm{Au}$ fil de ces arrêts, on voit donc que l'article 3 du règlement $\mathrm{n}^{\circ} 1$ est interprété de manière pragmatique, de sorte que les exigences de traduction qu'il pose ne soient pas disproportionnées par rapport aux enjeux réels. La traduction redondante n'a pas d'effet juridique et l'absence d'une traduction en principe exigée n'est sanctionnée que dans le cas où elle aurait pu conduire à une décision ou à un texte final différent du texte adopté.

20 TPICE, 3 mai 2007, Espagne c. Commission, T-219/04, Rec., p. II-1323, note E. Meisse, Europe, 2007 n $^{\circ}$ 7, comm. 175.

21 TPICE, 3 mai 2007, Espagne c. Commission, point 41. 
C'est encore l'enjeu de l'absence d'une traduction qui a donné lieu à une jurisprudence relative aux modalités de recrutement des fonctionnaires. En l'espèce, il s'agissait de savoir si des avis de vacances pour des postes à la Commission pouvaient n'être publiés que dans certaines langues officielles. Dans un arrêt Italie c. Commission, le Tribunal a jugé qu'une telle publication sélective ne pouvait pas constituer une violation du règlement $\mathrm{n}^{\circ} 1$, car celui-ci ne régit pas les relations entre les institutions et leurs fonctionnaires et agents. Le champ d'application de ce règlement est limité au régime linguistique applicable entre les institutions de la Communauté européenne et un État membre ou une personne relevant de la juridiction de l'un des États membres ${ }^{22}$. En l'absence de texte spécifique, le Tribunal a principalement égard à l'incidence de l'absence de traduction litigieuse. Il constate que celle-ci peut faire perdre une chance d'être recrutées à des personnes qui seraient recevables à poser leur candidature tout en n'ayant pas de connaissance des langues dans lesquelles l'avis de vacance est publié. Le Tribunal en conclut que la publication sélective de l'avis de vacances dans quelques langues seulement, «est susceptible de conduire, en l'absence d'autres mesures visant à permettre à cette dernière catégorie de candidats potentiels de prendre utilement connaissance du contenu de cet avis, à une discrimination à leur détriment ${ }^{23}{ }^{2}$. Là encore, c'est donc le pragmatisme qui prévaut: si une information adéquate avait été insérée dans toutes les langues au Journal officiel, fut-ce sous forme d'une brève notice renvoyant à un avis détaillé en quelques langues seulement, la solution aurait sans doute été différente. Tout comme l'interprétation jurisprudentielle du règlement $\mathrm{n}^{\circ} 1$, la solution de l'arrêt Italie $c$. Commission paraît inspirée par le principe d'effet utile de la traduction, en ce sens que c'est uniquement lorsque la traduction manquante aurait pu avoir pour effet de conduire à un résultat différent que le défaut de traduction est sanctionné.

22 TPICE, 20 novembre 2008, Italie c. Commission, T-185/05, non encore publié au Recueil, point 117

23 Italie c. Commission, précité, point 135. 
Au-delà de ce principe implicite d'effet utile, d'autres principes généraux du droit communautaire sont explicitement à l'œuvre dans la jurisprudence sur le régime linguistique des institutions. Ils complètent le règlement $\mathrm{n}^{\circ} 1$ dont on a vu qu'il ne couvrait que certaines situations.

\section{B. Les principes généraux du droit communautaire: compléments au régime juridique des institutions}

Le régime linguistique des institutions a été complété par la jurisprudence de la Cour à l'aide de principes généraux du droit. Parmi ces principes, il convient de mentionner tout d'abord un absent: le principe d'égalité des langues (1). En effet, l'égalité des langues ne constitue pas, à ce stade, un principe général de droit communautaire. En revanche, ont bien le statut de principe général en matière d'emploi des langues le principe de non-discrimination (2) et celui de sécurité juridique (3), auxquels il faut ajouter le principe de coopération loyale (4).

\section{L’inexistence d'un principe général d'égalité des langues}

A s'en tenir au traité, on pourrait penser que les différentes langues de l'Union sont sur un pied d'égalité. En effet, l'article $314 \mathrm{CE}^{24}$, qui conclut les dispositions finales et le traité, précise que toutes langues dans lesquelles le traité a été rédigé (quatre à l'origine) font également foi ${ }^{25}$. Elles ne sont cependant pas les seules puisque, au fur et à mesure des adhésions, toutes les autres langues officielles de l'Union ont été ajoutées comme langues faisant également foi ${ }^{26}$.

24 Cet article disparaît du traité sur le fonctionnement de l'Union européenne et est remplacé en substance par le nouvel article 4 TUE.

25 L'article $314 \mathrm{CE}$, al. 1, dispose: «[1]e présent traité rédigé en un exemplaire unique, en langue allemande, en langue française, en langue italienne et en langue néerlandaise, les quatre textes faisant également foi, sera déposé dans les archives du gouvernement de la République italienne, qui remettra une copie certifiée conforme à chacun des gouvernements des autres États signataires. »

26 L'article 314, al. 2, tel que modifié en dernier lieu par les actes d'adhésion de la Bulgarie et de la Roumanie et qui figure dans un appendice au traité, et précise que, «[e]n vertu des traités d'adhésion, font également foi les versions du présent 
La jurisprudence de la Cour sur l'interprétation qu'il convient de donner à un acte dont les différentes versions linguistiques divergent pourrait également donner le sentiment d'une certaine égalité des langues en droit communautaire. Dans cette hypothèse en effet, la Cour exige, selon une jurisprudence constante, que le texte d'une disposition soit interprété et appliqué à la lumière des versions établies dans les autres langues officielles ${ }^{27}$. Aussi toutes les versions linguistiques doivent-elles être prises en considération de manière égale aux fins de l'interprétation. De même, la valeur d'une langue ne saurait varier en fonction de l'importance de la population des États membres qui pratique la langue en cause ${ }^{28}$.

Pour autant, cette égalité des langues dans l'interprétation n'est pas absolue. En effet, au cas où différentes versions d'un texte se contredisent, l'interprétation doit être faite en fonction de l'économie générale et de la finalité de la réglementation en cause ${ }^{29}$. Ainsi, certaines versions linguistiques peuvent-elles s'imposer par rapport à d'autres ${ }^{30}$. En outre, lorsqu'une décision individuelle est publiée au Journal officiel et, partant, est traduite dans toutes les autres langues en vue d'informer les citoyens, seule la langue utilisée dans le cadre de la procédure concernée fera foi et sera utilisée pour l'interprétation de cette décision ${ }^{31}$. On voit donc que l'égalité formelle des langues prévue par le traité est relativisée en matière d'interprétation de versions linguistiques divergentes d'un même acte.

Plus radicalement, la Cour a jugé dans l'arrêt Kik qu'il n'existait pas de principe général d'égalité des langues. Dans cet arrêt, la Cour a jugé que les différentes références à l'emploi des langues présentes

traité en langues anglaise, bulgare, danoise, espagnole, estonienne, finnoise, grecque, hongroise, irlandaise, lettone, lituanienne, maltaise, polonaise, portugaise, roumaine, slovaque, slovène, suédoise et tchèque ».

27 CJCE, 12 juillet 1979, Koschniske, 9/79, Rec., p. 2717, point 6; CJCE, 2 avril 1998, EMU Tobacco, C-296/95, Rec., p. 1605, point 36.

28 Arrêt EMU Tobacco, cité supra note 27, point 36.

29 CJCE, 5 décembre 1996, Merck, C-267/95 et C-268/95, Rec., p. I-6285, points 21 à 24.

30 Conclusions Kokott sous CJCE, 11 décembre 2007, Skoma-Lux, C-161/06, p. I10841, point 30 et jurisprudence citée.

31 CJCE, 9 septembre 2003, Kik, C-161/03 P, Rec., p. I-8283, point 87. 
dans le traité «ne [pouvaient] être considérées comme étant la manifestation d'un principe général de droit communautaire assurant à chaque citoyen le droit à ce que tout ce qui serait susceptible d'affecter ses intérêts soit rédigé dans sa langue en toutes circonstan$\operatorname{ces}^{32}$ ». En particulier, la Cour a précisé qu'aucun principe d'égalité des langues ne résultait de l'article $314 \mathrm{CE}^{33}$. En l'espèce, ces considérations ont conduit la Cour à valider le régime linguistique de l'Office de l'harmonisation dans le marché intérieur (OHMI), lequel privilégie l'emploi d'un nombre restreint de langues. Elle a jugé que le traitement «différencié » des langues dans ce régime était compatible avec les principes de proportionnalité et de non-discrimination ${ }^{34}$. Cette dernière précision n'est pas anodine, puisque le principe de nondiscrimination, contrairement au principe d'égalité des langues, est bien un principe général applicable en matière d'emploi des langues par les institutions.

\section{Le principe de non-discrimination}

Lorsque certains justiciables peuvent prendre connaissance de la législation communautaire dans leur langue et d'autres non, il en résulte une discrimination. En ce sens, le principe de non-discrimination est sous-jacent à la jurisprudence de la Cour qui conditionne l'opposabilité d'un acte communautaire à un opérateur au fait que cette norme ait été préalablement publiée dans la langue officielle de l'État membre où cet opérateur est établi.

Ainsi, la Cour a jugé qu'une norme communautaire ne devenait opposable à une entreprise que lorsque le numéro du Journal officiel dans lequel elle est publiée est effectivement disponible dans la langue de l'État membre dont relève cette entreprise ${ }^{35}$. Comme le remarque l'avocat général Kokott dans ses conclusions sous l'arrêt Skoma Lux, la Cour refuse ce faisant de poser des limites à l'égalité des langues,

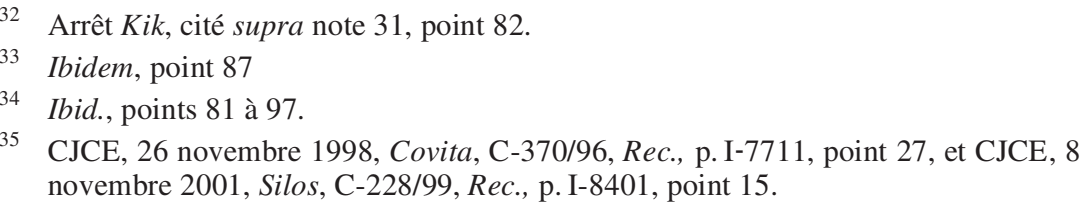


du moins pour les actes de portée générale ${ }^{36}$. Pour les actes de portée économique générale mais de nature non législative ou réglementaire, comme par exemple le cahier des charges d'une appellation d'origine protégée au niveau communautaire, la Cour s'est montrée attachée à une approche réaliste et empreinte d'un souci de sécurité juridique. Ainsi, elle a jugé qu'il n'était pas possible d'opposer à des opérateurs économiques ayant leur siège au Royaume-Uni certaines conditions du cahier des charges des appellations d'origine Prosciutto di Parma et Grana Padano, qui n'existaient qu'en italien ${ }^{37}$. Pour que ces conditions, comme l'interdiction de râper et d'emballer le fromage en dehors de la région d'origine, soient opposables à tous les opérateurs économiques, il faut qu'elles aient été portées à leur connaissance par une publicité adéquate dans la réglementation communautaire (par exemple une mention dans un règlement publié dans toutes les langues officielles) ${ }^{38}$.

Le principe de non-discrimination a trouvé un champ d'application nouveau avec les élargissements successifs. En effet, la publicité adéquate de l'ensemble de l'acquis communautaire dans les nouveaux États membres a constitué un véritable défi. Ainsi, lorsque, le $1^{\mathrm{er}}$ mai 2004, dix nouveaux États sont entrés dans l'Union européenne, des pans entiers de l'acquis communautaire n'avaient pu être publiés au Journal officiel de l'Union européenne dans les neuf nouvelles langues officielles. Les conséquences de l'absence de publication dans la langue officielle de ces nouveaux États membres d'un règlement communautaire sur les droits et obligations des opérateurs économiques ayant leur siège social dans ces États ont fait l'objet d'une demande de décision préjudicielle dans l'affaire Skoma-Lux ${ }^{39}$.

36 Conclusions Kokott sous CJCE, 11 décembre 2007, Skoma-Lux, C-161/06, Rec., p. I-10841.

37 CJCE, 20 mai 2003, Ravil, C-469/00, Rec., p. I-5053, points 99 et s., et CJCE, 20 mai 2003, Consorzio del Prosciutto di Parma et Salumificio S. Rita, C-108/01, Rec., p. I-5121, points 95 et s. Arrêts cités par l'avocat général Kokott, conclusions sous Skoma Lux, citées supra note 36, point 39.

38 Ibidem.

39 Cité supra note 36. 
Il ressort de l'arrêt rendu dans cette affaire qu'une réglementation non encore publiée dans le Journal officiel dans la langue officielle d'un nouvel État membre ne peut créer d'obligations à la charge des particuliers établis dans cet État, car cela constituerait une violation du principe d'égalité de traitement ${ }^{40}$. En effet, la solution contraire aurait pour effet de traiter de manière identique des situations objectivement différentes, à savoir, d'une part, celle des particuliers des nouveaux États membres, qui n'ont pas pu prendre connaissance de la réglementation dans la langue officielle de leur État membre et, d'autre part, celle les particuliers des anciens États membres, qui, eux, ont eu cette possibilité.

Certes, on pouvait objecter à cette approche formaliste que, en pratique, la plupart des justiciables prennent connaissance du droit communautaire non en lisant le Journal officiel, mais en consultant les sites internet officiels. La juridiction de renvoi n'avait du reste pas manqué de le relever ${ }^{41}$. Ainsi, était-il mis en avant que, même en l'absence de publication au Journal officiel dans leurs langues, les justiciables des nouveaux États membres avaient la possibilité de s'informer sur les réglementations en cause au moyen de traductions disponibles gratuitement sur le site internet EUR-Lex. Suivant les conclusions de l'avocat général Kokott, la Cour a cependant estimé que ces traductions officieuses ne constituaient pas une publicité adéquate au sens de sa jurisprudence ${ }^{42}$. En effet, la publication sur le site internet officiel de la Commission de versions linguistiques de la réglementation douanière en cause à l'usage des opérateurs des nouveaux États membres ne garantissait pas un degré de fiabilité équivalant à celui d'une version publiée en bonne et due forme au Journal officiel ${ }^{43}$. A cet égard, la Cour juge que, dans la mesure où le droit communautaire, à la différence des droits de certains États mem-

40 CJCE, 11 décembre 2007, Skoma-Lux, C-161/06, point 39. Voy. K. LASINSKISUlECKI et W. MORAWSKI, «Late Publication of EC Law in Languages of New Member States and its Effects: Obligations on Individuals Following the Court's Judgment in Skoma-Lux», C.M.L.R., vol. 45, 2008, p. 705-725.

41 Arrêt Skoma-Lux, cité supra note 36, point 19.

42 Jurisprudence citée supra note 37.

43 Arrêt Skoma-Lux, cité supra note 36, point 48; conclusions Kokott sous SkomaLux, citées supra note 36, point 66. 
bres, ne réglemente pas la publication de la législation sur internet, une telle publication n'est pas suffisante pour permettre son opposabilité à l'égard des justiciables ${ }^{44}$.

Dans le même ordre d'idées, la publication par les ministères concernés des règlements en cause dans les langues officielles des nouveaux États membres ne constitue pas non plus une publicité adéquate. La Cour justifie cette inopposabilité au motif que les règlements communautaires ne requièrent aucune mesure de réception dans le droit national ${ }^{45}$.

Au regard du principe de non-discrimination, le résultat de l'arrêt Skoma-Lux paraît quelque peu paradoxal. En effet, il résulte de l'arrêt que les entreprises établies dans les nouveaux États membres ne sont pas tenues de verser certains droits de douane alors que les entreprises établies dans les anciens États membres sont, elles, tenues de payer ces mêmes droits. Cette différence de traitement est justifiée, comme on l'a vu, au nom du principe de non-discrimination et, comme on le verra, au nom du principe de sécurité juridique. Pour s'en tenir ici au premier de ces principes, on peut observer que, pour éviter une discrimination juridique - au regard de l'accès au droit - la Cour consacre une discrimination économique - au regard de la fiscalité - entre entreprises qui se concurrencent dans le cadre d'un marché intérieur et d'une union douanière unique ${ }^{46}$. On peut également s'interroger sur l'incidence de la distinction entre établissement primaire et secondaire au regard de la solution consacrée. Ainsi, une entreprise établie à titre principal en Belgique ou en Allemagne et dont une filiale serait implantée en Pologne ou en République tchèque pourrait-elle invoquer la jurisprudence Skoma-Lux et échapper au versement de certains droits de douane au motif que sa filiale mène son activité économique depuis le territoire d'un État membre dans la langue duquel la réglementation communautaire n'a pas été publiée au Journal officiel? Sans doute pourrait-on invoquer à l'encontre d'une telle solution le principe général de prohibition de l'abus, mais la jurisprudence montre que la Cour

44 Arrêt Skoma-Lux, cité supra note 36, point 49.

45 Conclusions Kokott sous Skoma-Lux, citées supra note 36, points 53 et 54.

46 Voyez les critiques de K. LASINSKI-SUleCKI et W. MoraWSKI, cité supra note 40, p. 714-715. 
a, en matière de liberté d'établissement, une conception restrictive de l'abus ${ }^{47}$. Il n'est donc pas exclu que la jurisprudence Skoma-Lux, en sanctionnant une discrimination formelle n'aboutisse à ouvrir la porte à des discriminations réelles, voire à des stratégies de contournement de la législation non publiée dans toutes les langues de l'Union.

\section{Le principe de sécurité juridique}

La solution de l'arrêt Skoma-Lux s'appuie non seulement sur le principe de non-discrimination, mais aussi sur le principe de sécurité juridique. En effet, l'opposabilité des actes de droit communautaire aux personnes physiques et morales des nouveaux États ayant adhéré à l'UE en 2004 est subordonnée aux conditions générales de mise en œuvre du droit communautaire telles qu'elles sont prévues par les traités originaires. La Cour rattache cette obligation au principe de sécurité juridique, lequel «implique qu'une réglementation doit permettre aux intéressés de connaître avec exactitude l'étendue des obligations qu'elle leur impose ${ }^{48} »$. Ce raisonnement s'inscrit dans le prolongement d'une jurisprudence constante depuis 1979, qui conditionne l'opposabilité des actes de droit communautaire à la possibilité pour les justiciables d'en prendre connaissance ${ }^{49}$.

Le fait que, dans l'affaire Skoma-Lux, la question préjudicielle ait été posée dans le cadre d'une affaire pénale explique probablement l'interprétation stricte du principe de sécurité juridique retenue par la Cour, en écho sans doute à l'adage nullum crimen sine lege. En effet, dans cet arrêt, la Cour va jusqu'à faire prévaloir le principe de sécurité juridique sur l'effet utile du règlement douanier, dont l'application territoriale connaitra ainsi des lacunes.

On notera par ailleurs que les justiciables tchèques, qui ont vu reconnaître à leur profit l'inopposabilité du règlement communautaire en cause, n'obtinrent dans l'arrêt Skoma-Lux qu'une victoire à la

47 Voy. notamment: CJCE, 9 mars 1999, Centros, C-212/97, Rec., 1999, p. I-1459; CJCE, 5 juin 1997, VT4, C-56/96, Rec., p. I-3143.

48 Arrêt Skoma-Lux, cité supra note 36 point 38 ; conclusions Kokott, citées supra note 36 , point 36 .

49 CJCE, 25 janvier 1979, Racke, 98/78, Rec., p. 69, point 15. 
Pyrrhus. En effet, ce que la Cour a accordé d'une main (inopposabilité du règlement) au titre de la sécurité juridique, elle le reprend de l'autre, et ce en vertu du même principe. En effet, conformément à une jurisprudence complexe sur la validité des actes administratifs nationaux contraires au droit communautaire, la Cour a jugé in fine que les États membres concernés n'étaient pas tenus de remettre en cause des décisions administratives ou juridictionnelles prises sur la base de règlements communautaires non publiés dans le Journal officiel. Ce maintien d'actes nationaux illicites - en ce qu'ils sont fondés sur des règlements communautaires n'ayant pas été valablement publiés - se justifie au regard du principe de sécurité juridique. En d'autres termes, le principe de sécurité juridique implique l'inopposabilité du règlement douanier, mais pas l'obligation pour les États concernés de rembourser les droits de douane illégalement perçus.

Ce résultat paradoxal du maintien des décisions nationales n'est toutefois obtenu que si deux conditions sont remplies: en premier lieu, elles doivent être «devenues définitives en vertu des règles nationales applicables ${ }^{50} »$, en second lieu, elles ne doivent pas porter atteinte à des droits fondamentaux ${ }^{51}$. Comme la Cour renvoie cette appréciation au juge national, l'arrêt ne dit pas si la protection de la propriété privée aurait pu jouer. En l'espèce, la Cour n'a pas non plus exigé le respect du principe d'effectivité et d'équivalence par les règles nationales susceptibles de conférer un caractère définitif aux décisions contestées ${ }^{52}$.

Outre les principes de non-discrimination et de sécurité juridique, la jurisprudence de la Cour en matière d'exigences de traduction s'appuie aussi sur le principe de coopération loyale, comme l'illustre encore l'arrêt Skoma-Lux.

Arrêt Skoma-Lux, cité supra note 36, point 72.

Ibidem, point 73 .

52 CJCE, 13 janvier 2004, Kühne et Heitz, C-453/00, Rec., I-837. Y. HOUYET, «Le rétablissement de la légalité communautaire par le retrait d'actes administratifs nationaux », J.D.E., 2008, n 33. 


\section{Le principe de coopération loyale}

Découlant de l'article $10 \mathrm{CE}^{53}$, le principe de coopération loyale oblige les États membres à prendre toutes les mesures pour garantir l'effectivité du droit communautaire dans leur ordre juridique. Dans l'arrêt Skoma-Lux, la Cour énonce une limite à ce principe en jugeant qu'il n'a pas pour effet de permettre aux nouveaux États membres qui ont adhéré à l'UE en 2004 d'opposer à leurs justiciables des règlements communautaires qui n'ont pas été publiés au Journal officiel de l'Union européenne dans leur langue officielle ${ }^{54}$. Il ne revient pas, en effet, aux États membres de faire supporter aux justiciables, les conséquences négatives découlant du non-respect de l'obligation de publication qui pesait à l'origine sur l'administration communautaire ${ }^{55}$. En d'autres termes, la sanction de l'absence de l'absence de traduction de la législation ne doit pas atteindre les particuliers.

Au terme de ce bref survol, on prendra la mesure, d'une part, de l'hétérogénéité des sources du régime linguistique des institutions et, d'autre part, de ses lacunes. Ainsi, le règlement $\mathrm{n}^{\circ} 1 \mathrm{n}$ 'a été mis à jour qu'a minima alors que les relations entre les particuliers et les institutions se sont complexifiées au fil des décennies. Il est frappant de constater que le régime linguistique des institutions, qui paraissait devoir être fixé dès l'origine est peu développé sur le plan réglementaire. Le règlement $n^{\circ} 1$, très bref, n'a été mis à jour qu'à travers l'ajout mécanique des langues officielles des nouveaux États membres. Il résulte de cette immobilité du droit écrit, d'une part, sur le plan pratique, un écart grandissant entre des dispositions lacunaires et une pratique institutionnelle qui rationnalise l'emploi des langues, et d'autre part, sur le plan des sources du droit communautaire applicable aux obligations de traduction des institutions, une grande place laissée à la jurisprudence et aux principes généraux du droit.

On ne peut toutefois s'arrêter à ces constats si l'on veut prendre la mesure du droit européen de la traduction. En effet, la construction

\footnotetext{
53 Cet article disparaît du nouveau traité sur le fonctionnement de l'Union européenne et est repris en substance à l'article 4 du traité UE modifié.

54 Arrêt Skoma-Lux, cité supra note 36, point 41.

55 Ibidem, point 42.
} 
européenne ne se limite pas à ses institutions et les questions relatives à l'emploi des langues, et donc aux obligations de traduction, ne concernent pas seulement les relations entre le législateur et les justiciables ni, plus généralement, les relations entre les institutions et les particuliers. Au contraire, un grand nombre de questions linguistiques apparaissent dans le fonctionnement ordinaire du marché intérieur ou de l'espace de liberté, de sécurité et de justice. C'est pourquoi il convient d'analyser les exigences linguistiques posées ou permises par le droit communautaire à l'aune des libertés économiques et en matière de coopération judiciaire civile.

\section{Les exigences linguistiques au prisme des libertés économiques}

En droit communautaire, les libertés de circulation ont le statut de libertés fondamentales. La libre circulation des marchandises (articles 28 à $30 \mathrm{CE}$, articles 34 à $36 \mathrm{TFUE}$ ), des travailleurs (article $39 \mathrm{CE}$, article 45 TFUE) et la libre prestation de services (article $49 \mathrm{CE}$, article 56 TFUE) constituent en effet les piliers du marché intérieur ${ }^{56}$. Au regard de ces libertés, les exigences relatives à l'emploi des langues apparaissent ambivalentes. En effet, la traduction apparaît tour à tour comme une nécessité pour le bon fonctionnement du marché et comme un obstacle aux libertés de circulation. Plus précisément, la traduction de certains documents (par exemple, des offres commerciales ou informations destinées aux clients) relève de la simple nécessité commerciale lorsqu'ils sont destinés à des consommateurs d'un État membre autre que celui dans lequel est établie l'entreprise qui en est l'auteur. En d'autres termes, le marché intérieur ne saurait être effectif dans un contexte multilingue si les entreprises ne faisaient pas l'effort de s'adresser à leurs fournisseurs ou à leurs clients dans une langue qu'ils comprennent. Il en va de même pour la communication entre les particuliers et les autorités publiques des différents États membres dans lesquels ils exercent leur activité. En ce sens donc, l'exercice par les particuliers de l'une des libertés de circulation qui sont garanties à

56 Il ne sera pas question de la libre circulation des capitaux dans la présente contribution. 
leur profit par le traité suppose une activité de traduction. Mais ni les droits nationaux ni le droit communautaire ne s'en remet aux particuliers pour déterminer l'étendue de cette activité. Celle-ci est au contraire encadrée, tant par des dispositions nationales que par des dispositions communautaires. A titre d'exemple, une réglementation allemande prévoit que les entreprises présentes sur le territoire allemand doivent être en mesure de fournir aux inspecteurs du travail des contrats de travail rédigés en langue allemande, et ce alors même que les salariés n'effectuent qu'une mission temporaire en Allemagne ${ }^{57}$. Parallèlement, le droit communautaire, au nom de l'objectif de protection des consommateurs, prévoit diverses obligations d'étiquetage, qui supposent pour les importateurs de traduire les mentions figurant sur les emballages des marchandises qu'ils commercialisent. Si ces différentes obligations de traduction s'expliquent par des considérations légitimes de protection des consommateurs, des salariés, voire simplement de protection ou de promotion d'une langue nationale, il n'en reste pas moins qu'elles rendent plus difficiles ou plus coûteuses les activités transfrontalières. En ce sens, les obligations de traduction ne facilitent pas l'exercice des libertés de circulation et y font au contraire potentiellement obstacle ${ }^{58}$. Au sein du marché intérieur, la traduction apparaît donc à la fois comme un adjuvant et comme un obstacle aux libertés de circulation.

Parce que les règlementations nationales sur l'usage des langues peuvent entraver l'exercice des libertés de circulation, elles sont encadrées par le droit communautaire. Celui-ci procède de deux manières. La première est l'harmonisation. Elle consiste à définir, généralement par voie de directives, un certain nombre des exigences harmonisées (par exemple en ce qui concerne l'étiquetage de certains produits). Ainsi, les réglementations des États membres poursuivant des objectifs similaires (par exemple la protection des consommateurs) mais adoptant des modalités différentes (par exemple des mentions obligatoires qui ne sont pas les mêmes) ne peuvent plus être trop divergentes

V. infra D.

58 Voy. sur ce thème l'étude très complète de M. CANDELA-SoRIANO, «Les exigences linguistiques : une entrave légitime à la libre circulation?», $C D E, 2002$, p. 944. 
ni, partant, créer d'obstacles significatifs à la libre circulation des marchandises. En présence d'une harmonisation, les exigences linguistiques que peuvent imposer les États seront soumises à un contrôle de conformité avec la règle harmonisée. En l'absence d'harmonisation particulière dans un domaine donné, les règles relatives à l'emploi des langues sont également contrôlées, mais cette fois au regard des libertés fondamentales garanties par le traité. A cet égard, on verra que peuvent être sanctionnées non seulement les règles d'origine publique qui créent des obstacles à la libre circulation, mais également des exigences privées ${ }^{59}$.

Ce double encadrement par le droit dérivé et par le traité peut être illustré dans le champ des différentes libertés: libre circulation des marchandises (A), libre circulation des travailleurs (B), liberté d'établissement (C) et libre prestations de services (D).

\section{A. Traduction et libre circulation des marchandises: des étiquettes et des mots réglementés}

Dans le champ de la libre circulation des marchandises, on trouve une forte harmonisation. La réglementation communautaire ayant une incidence sur l'emploi des langues est abondante et variée et c'est au regard de ce droit dérivé que les exigences linguistiques nationales sont contrôlées. A titre d'illustration, on peut évoquer les règles sur l'étiquetage des produits ainsi que certaines applications du droit des marques.

En matière d'étiquetage des produits, il existe une réglementation assez ancienne. L'une des directives de rapprochement des législations nationales porte sur l'étiquetage des denrées alimentaires ${ }^{60}$. Cette directive définit les mentions informatives qui doivent obligatoirement

59 V. infra les développements consacrés à l'arrêt Angonese.

60 Directive 79/112/CEE du Conseil, du 18 décembre 1978, relative au rapprochement des législations des États membres concernant l'étiquetage et la présentation des denrées alimentaires destinées au consommateur final ainsi que la publicité faite à leur égard, $J O 1979$, L 33, p. 1. Cette directive est aujourd'hui remplacée par la directive 2000/13/CE, relative au rapprochement des législations des États membres concernant l'étiquetage et la présentation des denrées alimentaires ainsi que la publicité faite à leur égard, $J O$ L 109 p. 29. 
figurer sur les emballages alimentaires et prévoit que celles-ci doivent être libellées «dans une langue facilement comprise par les acheteurs, sauf si l'information de l'acheteur est assurée par d'autres mesures ${ }^{61} \gg$. Cette formule vague, qui s'explique peut être par le souci de concilier la libre circulation des marchandises et la protection des consommateurs n'a pas manqué de donner lieu à un contentieux, illustré par l'affaire Piageme ${ }^{62}$.

Cette affaire concernait une réglementation belge prévoyant que les étiquettes sur certaines denrées devaient être libellées dans la langue de la région linguistique de commercialisation. Dans son premier arrêt Piageme, la Cour a constaté que cette réglementation nationale était plus stricte que ce qu'autorisait la directive ${ }^{63}$. En outre, elle méconnaissait la possibilité pour les entreprises d'assurer l'information des consommateurs par d'autres moyens que l'étiquetage. La Cour juge donc la règle nationale contraire à la directive et fait en conséquence prévaloir l'exigence de libre circulation des marchandises sur une politique régionale relative à l'emploi des langues et sur la protection des consommateurs. Cet arrêt indique donc que, en présence d'une législation harmonisée, une exigence nationale de traduction trop stricte doit être supprimée. C'est du reste la mise en ouvre de cette solution qui a donné lieu au second arrêt Piageme ${ }^{64}$.

Si dans l'affaire Piageme, la réglementation en cause avait pour objet la protection des langues régionales, tel n'est pas toujours le cas. Il arrive en effet que des réglementations nationales sur l'étiquetage n'aient que fortuitement une incidence linguistique, par exemple lorsqu'elles viennent à s'appliquer à des marchandises originellement commercialisées dans une autre langue. C'est ce qu'illustre un arrêt Commission c. Espagne ${ }^{65}$. Était en l'espèce en cause une réglementation espagnole permettant l'utilisation du terme «biológico» ou «bio» pour des produits non-issus d'un mode de production biologi-

61 Article 14 de la directive 79/112/CEE citée supra note 60.

62 CJCE, 18 juin 1991, Piageme c. Peeters, C-369/89, Rec., p. I-2971 et CJCE, 12 octobre1995, Piageme c. Peeters, C-85/94, Rec., p. I-2955.

63 Arrêt Piageme I, cité supra note 62, point 16.

64 Arrêt Piageme II, cité supra note 62.

65 CJCE, 14 juillet 2005, Commission c. Espagne, C-135/03, Rec., p. I-6909. 
que. Cette permissivité allait naturellement à l'encontre des intérêts des fabricants de produits réellement issus d'un mode de production respectueux de l'environnement et plusieurs d'entre eux avaient saisi la Commission de plaintes, arguant que la réglementation espagnole était contraire à un règlement communautaire relatif à l'étiquetage et au contrôle des produits issus du mode de production biologique ${ }^{66}$. S'agissant cette fois d'un règlement et non d'une directive de rapprochement des législations, la marge de manœuvre des autorités nationales était inexistante. La situation devait être appréciée directement à l'aune du règlement communautaire. Malheureusement pour ces plaignants, le terme espagnol dont l'usage est encadré par ce règlement était «ecológico» et non «biológico». Partant, la réglementation espagnole ne violait pas le droit communautaire en laissant libre l'usage de ce dernier terme. Dans cette affaire, la question de savoir si, pour des consommateurs de langue espagnole, ces deux termes sont ou non synonymes avait été débattue. Quelle que soit la réponse apportée à cette question, le simple fait que le risque de confusion soit manifeste pour des entreprises ou des consommateurs non hispanisants illustre la nécessité pour le législateur communautaire de tenir compte du sens et des connotations d'un mot non seulement dans chaque langue officielle, mais aussi de la perception probable d'un mot réglementé par des locuteurs non natifs. Une difficulté de même ordre fut soumise à la Cour dans une affaire de marques.

L'affaire Matratzen Concord concernait la validité d'une marque enregistrée en Espagne par une entreprise faisant commerce de matelas ${ }^{67}$. La marque en cause se composait du nom Matratzen, qui signifie «matelas» en allemand. C'est précisément ce qui expliquait qu' un importateur allemand de matelas dont le nom commercial comportait également le mot Matratzen avait formé un recours en annulation

66 Règlement (CEE) n ${ }^{\circ}$ 2092/91 du Conseil du 24 juin 1991 concernant le mode de production biologique de produits agricoles et sa présentation sur les produits agricoles et les denrées alimentaires $(J O$ L 198, p. 1), modifié par le règlement (CE) n ${ }^{\circ}$ 1935/95 du Conseil, du 22 juin 1995 (JO L 186, p. 1), et par le règlement (CE) $\mathrm{n}^{\circ}$ 1804/1999 du Conseil, du 19 juillet 1999 (JO L 222, p. 1).

67 CJCE, 9 mars 2006, Matratzen Concord, C-421/04, Rec., p. I-2303. 
contre cette marque espagnole. La juridiction espagnole saisie du litige au principal se demandait si, bien qu'autorisé par le droit espagnol, l'enregistrement comme marque verbale nationale d'un terme générique issu d'une autre langue n'était pas contraire à la libre circulation des marchandises. Elle soulignait en effet que «les dénominations génériques appartenant aux langues des États membres doivent rester disponibles pour pouvoir être utilisées par n'importe quel entreprise établie dans ces États ${ }^{68}{ } \gg$. Le juge espagnol considérait plus précisément que «la marque espagnole Matratzen confère à son titulaire une position qui peut lui servir à limiter ou à restreindre l'importation de matelas en provenance d'États membres de langue allemande et, partant, à empêcher la libre circulation des marchandises ${ }^{69}{ }^{»}$.

Pour une fois, la Cour se montre moins intégrationniste que le juge national et juge ces scrupules non fondés. Il convient cependant de souligner que cette solution n'est pas acquise au regard du droit commun de la libre circulation des marchandises (articles 28 et $30 \mathrm{CE}$ visés dans la question préjudicielle), mais au regard du droit spécial que constitue la première directive sur les marques, laquelle harmonise de manière exhaustive les motifs d'invalidité d'une marque nationale $^{70}$. La Cour relève que, si la directive prévoit l'obligation de refuser l'enregistrement ou de prononcer la nullité d'une marque dépourvue de signe distinctif ou purement descriptive d'un produit ou service, elle ne contient en revanche aucune disposition permettant de refuser «les marques consistant dans un vocable emprunté à la langue d'un État membre autre que l'État d'enregistrement ${ }^{71} \gg$. Se référant à sa jurisprudence, la Cour ajoute que les motifs de refus et de nullité expressément visés par la directive doivent être appréciés en tenant compte de «la perception des milieux intéressés, à savoir dans le commerce et/ou chez le consommateur moyen desdits produits ou services, normalement informé et raisonnablement attentif et avisé,

68 Matratzen Concord, précité, point 13.

69 Ibidem, point 14.

70 Directive 89/104/CEE du Conseil, du 21 décembre 1988, rapprochant les législations des États membres sur les marques, JO 1989, L 40, p. 1, article 3. Voir sur ce point les conclusions de l'avocat général Jacobs sous l'arrêt Matratzen Concord, précité, point 40.

71 Matratzen Concord, précité, point 22. 
dans le territoire pour lequel l'enregistrement est demandé ${ }^{72} » . \mathrm{Ce}$ standard, à l'aune duquel les motifs de refus doivent être appréciés appellerait bien des remarques, mais il suffit ici de souligner que le consommateur moyen auquel la Cour fait référence est un consommateur national. En l'espèce, la question à laquelle doit répondre la juridiction nationale consiste à se demander si, pour le consommateur de matelas moyen en Espagne, le terme Matratzen est générique ou purement descriptif. Ce n'est qu'en cas de réponse affirmative à l'une ou l'autre de ces questions que la marque peut et doit être annulée.

On peut remarquer que la Cour, sans trancher cette question de fait, semble sceptique, comme l'indique la formulation restrictive qu'elle choisit: «La directive ne s'oppose pas à l'enregistrement dans un État membre, en tant que marque nationale, d'un vocable emprunté à la langue d'un autre État membre dans laquelle il est dépourvu de caractère distinctif ou est descriptif des produits ou des services pour lesquels l'enregistrement est demandé, à moins que les milieux intéressés dans l'État membre dans lequel l'enregistrement est demandé soient aptes à identifier la signification de ce vocable ${ }^{73}$.» Cette formule qui met l'accent sur l'incompétence linguistique probable du consommateur moyen contraste avec les conclusions de l'avocat général Jacobs, qui soulignait au contraire dans ses conclusions que «sur un marché de 452 millions de consommateurs, dont on peut raisonnablement s'attendre à ce que beaucoup d'entre eux comprennent d'autres langues que celles principalement parlées dans l'État membre où ils résident, l'autorité nationale compétente en matière de marques doit être particulièrement consciencieuse lorsqu'elle décide si un signe constitué d'un terme étranger désignant ou décrivant les produits en cause peut être enregistré ${ }^{74} \gg$. En ce sens, on ne peut s'empêcher de penser que, à travers son arrêt Matratzen Concord, la Cour semble indiquer aux opérateurs privés que les comportements qui s'appuient sur la diversité linguistique de l'Union pour ériger des barrières d'ordre

\footnotetext{
Matratzen Concord, point 24.

Ibidem, précité, point 26. Nous soulignons.

74 Conclusions Jacobs sous Matratzen Concord, précitées, point 54.
} 
privé à la libre circulation des marchandises pourraient ne pas être très sévèrement surveillés.

De telles barrières peuvent également contrarier la libre circulation des travailleurs, surtout lorsqu'elles sont renforcées par des règles qui visent à promouvoir une langue nationale.

\section{B. Langues et libre circulation des travailleurs : des connaissances linguistiques des employés}

Dans le domaine de la libre circulation des travailleurs, on trouve un double encadrement des exigences linguistiques nationales par le droit communautaire selon le schéma déjà évoqué. Il existe, d'une part, certaines mesures d'harmonisation et, d'autre part, en dehors du domaine harmonisé, un contrôle des exigences nationales de traduction au regard des règles du traité. Il convient par ailleurs de souligner une particularité, puisque, en matière de libre circulation des travailleurs, la Cour a eu l'occasion de se prononcer non seulement sur des règles linguistiques d'origine publique, mais également sur des pratiques privées.

En matière d'accès à un emploi public, la Cour a été amenée à se prononcer sur la compatibilité avec le droit communautaire d'exigences de connaissance de la langue nationale. Ainsi, dans un arrêt Groener $^{75}$, était en cause la légalité d'une exigence imposée en République d'Irlande aux candidats à un poste de professeur dans l'enseignement public. Ceux-ci devaient démontrer leur connaissance de l'irlandais, quand bien même cette connaissance n'était pas indispensable à l'exercice de leur activité (la plupart des cours étant donnés en anglais). Il n'était pas contestable qu'une telle exigence créait un obstacle à la libre circulation des travailleurs. La question était donc de savoir si elle pouvait néanmoins être justifiée. La Cour a jugé que tel était le cas, à condition que cette exigence soit appliquée de manière non discriminatoire et proportionnée. En admettant qu'une exigence linguistique puisse être conforme au droit communautaire alors même qu'elle n'est pas justifiée au regard des tâches spécifiques de l'emploi, la Cour fait clairement prévaloir la politique nationale de

75 CJCE, 8 novembre 1989, Groener, C-379/87, Rec., p. 3967. 
sauvegarde du patrimoine linguistique sur la libre circulation des travailleurs. Le fait qu'il s'agisse d'une politique publique explique peutêtre cette faveur, car on ne la retrouve pas lorsqu'il s'agit d'une pratique privée.

En matière d'accès à un emploi privé, la Cour a été saisie dans l'affaire Angonese de la pratique d'une banque italienne qui, pour recruter les employés d'un de ses établissements, situé à Bolzano dans la région bilingue frontalière avec l'Autriche, exigeait qu'ils réussissent un concours ${ }^{76}$. Ce point ne posait aucune difficulté en lui-même. En revanche, il n'en allait pas de même de l'une des conditions d'accès à ce concours de recrutement, lequel était limité aux seuls détenteurs d'un certificat de bilinguisme ne pouvant être obtenu que localement. Il y avait là une discrimination indirecte à raison de la nationalité, dans la mesure où il était plus difficile pour un candidat non issu de la province de Bolzano d'obtenir ce certificat que pour un candidat local ${ }^{77}$. La Cour précise que, à cet égard, il importe peu que la discrimination frappe non seulement les travailleurs des autres États membres, mais également les candidats issus d'autres provinces italiennes; il suffit, pour que la situation tombe sous le coup du principe de non-discrimination que l'accès à l'emploi soit rendu plus difficile pour les ressortissants d'autres États membres ${ }^{78}$. Ce qui est condamné en l'espèce n'est bien sûr pas l'exigence de bilinguisme imposée par la banque à ses futurs employés, mais les modalités très restrictives admises par l'employeur pour prouver ce bilinguisme. En somme, la Cour dans cet arrêt dégage un principe de liberté de la preuve au bénéfice des travailleurs devant établir leurs compétences linguistiques pour accéder à un emploi privé.

On trouve également des déboires linguistiques en matière d'accès à une profession réglementée, ce qui relève de la liberté d'établissement.

6 CJCE, 6 juin 2000, Angonese, C-281/98, Rec., p. I-4139.

77 Angonese, points 39-41.

78 Angonese, point 41. 


\section{Langues et liberté d'établissement: des professionnels ignorant la langue nationale}

Dans le domaine de la liberté d'établissement, il existe, comme en matière de libre circulation des marchandises, une importante œuvre d'harmonisation. De nombreuses directives visent à faciliter l'accès à diverses professions ou l'exercice de certaines activités réglementées. Ainsi les ordres professionnels ou autres organismes chargés de définir au niveau national les conditions d'accès à une profession, y compris les exigences linguistiques, voient-ils leurs pratiques encadrées par le droit communautaire.

L'ordre du barreau de Luxembourg en a fait l'expérience, comme en témoigne l'arrêt Wilson ${ }^{79}$. En l'espèce, M. Wilson, ressortissant britannique établi au Luxembourg, souhaitait y pratiquer la profession d'avocat sous son titre professionnel d'origine, à savoir celui de barrister. La directive sur l'établissement des avocats prévoit cette possibilité et précise que l'avocat migrant doit s'inscrire auprès de l'autorité compétente dans l'État membre d'établissement ${ }^{80}$. Ce texte ajoute que cette autorité «procède à l'inscription de l'avocat au vu de l'attestation de son inscription auprès de l'autorité compétente de l'État membre d'origine ${ }^{81}$ ». La législation luxembourgeoise prévoyait cependant que les avocats voulant s'inscrire au barreau de Luxembourg pour pratiquer sous leur titre d'origine devaient se soumettre à une vérification orale de leurs connaissances linguistiques en français, en allemand et en luxembourgeois. C'est cette exigence linguistique que contestait $\mathrm{M}$. Wilson, en arguant de sa contrariété avec la directive relative à la liberté d'établissement des avocats.

La Cour lui donna raison et dit pour droit que la directive «n'admet [...] pas que l'inscription d'un avocat européen auprès de l'autorité compétente de l'État membre d'accueil puisse être subordonnée à un entretien censé permettre à ladite autorité d'évaluer la

79 CJCE, 19 septembre 2006, Wilson c. Luxembourg, C-506/04, Rec., p. I-8613.

80 Directive 98/6/CE visant à faciliter l'exercice permanent de la profession d'avocat dans un État membre autre que celui où la qualification a été acquise, $J O \mathrm{~L} 77$, p. 36, articles 2 et 3 .

81 Directive 98/6/CE citée supra note 80, article 3, paragraphe 2. 
maîtrise, par l'intéressé, des langues de cet État membre ${ }^{82}{ }$. La solution était techniquement prévisible en ce que la Cour refuse à un État membre le droit de réintroduire des exigences restreignant une liberté de circulation dans un domaine harmonisé. Du point de vue de l'emploi des langues, elle signifie qu'un avocat inscrit dans un État membre peut pratiquer sa profession dans un autre État membre non seulement sous son titre d'origine, mais encore dans sa langue d'origine. La Cour relève à cet égard, que la protection des consommateurs de services juridiques ne saurait justifier une solution différente. En effet, l'avocat exerçant sous son titre d'origine plutôt que sous le titre de l'État d'établissement signale par ce seul fait qu'il n'est pas pleinement intégré dans la profession au sein de cet État. En outre, en permettant l'exercice sous le titre d'origine, la directive a entendu «répondre [...] aux besoins des usagers du droit, lesquels, en raison des flux d'affaires croissants résultant notamment du marché intérieur, recherchent des conseils lors de transactions transfrontalières dans lesquelles sont souvent imbriqués le droit international, le droit communautaire et les droits nationaux ${ }^{83} \gg$. La Cour précise que, pour ce type d'affaires, la diversité linguistique d'un barreau est plutôt un atout. Elle note en effet que «[d]e tels dossiers internationaux, de même que des affaires relevant du droit d'un État membre autre que l'État membre d'accueil, peuvent ne pas nécessiter un degré de connaissance des langues de ce dernier État membre aussi élevé que celui requis pour le traitement de dossiers dans lesquels le droit de cet État membre est applicable». Les exigences linguistiques comme celles imposées au Luxembourg aux avocats étrangers sont donc non seulement interdites au titre de la directive applicable, mais encore, et plus généralement, non fondées en ce qu'elles portent atteinte à une richesse linguistique de nature à répondre à des besoins spécifiques dans le marché commun. A travers la motivation de l'arrêt Wilson apparaît donc en quelque sorte un argument économique en faveur de la diversité linguistique. Celui-ci est distinct de l'argument qui peut être tiré de l'article 22 de la Charte des droits fondamentaux, aux ter-

82 Wilson, cité supra note 79 , point 77.
83 Wilson, cité supra note 79 , point 75. 
mes duquel «[1]'Union respecte la diversité culturelle, religieuse et linguistique». En effet, il ne s'agit pas ici de respecter la diversité linguistique protégée par les États membres - les autorités luxembourgeoises, à travers la réglementation en cause dans l'arrêt Wilson cherchaient peut-être précisément à protéger l'usage du luxembourgeois dans les professions juridiques. La maxime qui s'évince de l'arrêt Wilson serait plutôt celle-ci : l'Union favorise la diversité linguistique. Cet arrêt illustre donc que respecter et favoriser la diversité linguistique peuvent constituer deux objectifs distincts.

Pour autant, la protection des langues dans leur intégrité n'est pas absente de la jurisprudence de la Cour, même en matière économique. La Cour a eu l'occasion d'en juger dans le domaine de la libre prestation de services, où sa jurisprudence illustre également la possible convergence entre la protection par les États membres de l'emploi de leur langue nationale et la protection des travailleurs.

\section{Transcription, traduction et libre prestation de services}

L'intégrité d'une langue n'est pas toujours protégée uniquement dans l'État où elle est parlée. Le droit communautaire conduit parfois à étendre cette protection à d'autres États membres, ainsi que l'illustre un arrêt Konstantinidis ${ }^{84}$. Dans cette affaire, la Cour est amenée à protéger, au nom de la libre prestation de services, la translittération fidèle d'un nom grec en caractères latins dans les registres d'état civil en Allemagne. En l'espèce, un ressortissant grec établi en Allemagne avait vu transcrire son nom dans les registres de l'état civil selon une graphie qui n'était pas conforme à la prononciation de son nom en grec. La transcription qu'il jugeait fautive avait pourtant été faite conformément à la réglementation allemande qui renvoyait à une norme ISO, ainsi qu'à la jurisprudence des cours allemandes. C'est pourquoi il n'avait pu obtenir des autorités compétentes la rectification de «Konstadinidis» (transcription contestée) en «Konstantinidis (transcription souhaitée) ${ }^{85}$. Le litige avait acquis une dimension communautaire, car l'intéressé exerçait à la profession de masseur et

84 CJCE, 30 mars 1993, Konstantinidis, C-168/91, Rec., p. I-1191.

85 Konstantinidis, cité supra note 84, point 5. 
d'assistant en hydrothérapie. Il était donc prestataire de services au sens du traité. A ce titre, il faisait valoir que la translittération erronée de son patronyme lui causait un préjudice, car elle conduisait à en altérer la prononciation de manière telle que cela risquerait de créer une confusion dans l'esprit des clients potentiels. C'est l'argument qu'a retenu la Cour pour condamner l'entrave potentielle à la libre prestation de services. Ainsi, le droit du marché intérieur peut conduire à forcer les États membres à respecter l'intégrité linguistique du nom d'un acteur économique.

C'est encore pour protéger un intérêt privé, mais cette fois collectif puisqu'il s'agit de l'intérêt des travailleurs, que la Cour a été amené à se prononcer en faveur d'une exigence relative à l'emploi d'une langue dans la sphère économique. Dans un arrêt Commission c. Allemagne ${ }^{86}$, était en cause une réglementation allemande qui faisait obligation à toute entreprise employant des travailleurs sur des chantiers situés en Allemagne de tenir à la disposition des autorités de contrôle pour chaque ouvrier une version en langue allemande de son contrat de travail, des fiches de paie et documents attestant des horaires de travail et du paiement des salaires. Pour les entreprises établies dans d'autres États membres cette obligation représentait indéniablement un coût, puisqu'elle les obligeait à traduire en allemand des documents établis à l'origine dans une autre langue. Il y avait donc une discrimination indirecte, en ce qu'étaient imposés aux entreprises établies dans un État membre non germanophone des coûts administratifs que les entreprises allemandes n'avaient pas à supporter. En ce sens, la réglementation allemande constituait une entrave à la libre prestation de services ${ }^{87}$. La Cour juge toutefois cette restriction justifiée au regard de l'exigence impérative d'intérêt général que constitue la protection des travailleurs. Plus précisément, il s'agit de garantir aux travailleurs détachés sur des chantiers situés en Allemagne par des entreprises établies dans d'autres États membres la protection offerte par le droit allemand. A cet égard, la Cour juge l'exigence de traduc-

86 CJCE, 18 juillet 2007, Commission c. Allemagne, C-490/04, p. I-6095.

87 Commission c. Allemagne, cité supra note 86, points 68 et 69 . 
tion en allemand des contrats de travail et autres documents conforme au principe de proportionnalité. Elle relève en effet que l'obligation de traduction ne concerne qu'un nombre limité de documents standardisés et il n'existe pas de moyens moins contraignants de permettre le contrôle du respect de la législation sociale par les inspecteurs du travail allemands.

Au terme d'un contrôle de proportionnalité, la Cour fait donc prévaloir, au nom de la protection des travailleurs détachés, une exigence linguistique nationale sur la libre prestation de services. La différence entre cette solution et celle de l'arrêt Wilson souligne le rôle que joue dans le raisonnement de la Cour l'objectif poursuivi par la réglementation nationale protectrice de l'usage d'une langue. La réglementation luxembourgeoise sur les avocats apparaissait protectionniste dans la mesure où elle n'était pas réellement justifiée par l'intérêt des consommateurs de services juridiques, tandis que la réglementation allemande apparaît justifiée, car elle vise à protéger les travailleurs détachés et poursuit cet objectif légitime par des moyens proportionnés. La comparaison avec l'arrêt Groener évoqué plus haut concernant les exigences linguistiques irlandaises est plus délicate, car l'arrêt Commission c. Allemagne a été rendu dans le cadre d'une procédure en manquement, ce qui autorise la Cour à procéder elle-même au contrôle de proportionnalité, tandis que, saisie dans l'affaire Groener d'une question préjudicielle, elle avait pu laisser au juge national le soin de trancher ce point. La question de savoir si le droit communautaire, dans sa dimension économique, est plus favorable à certaines justifications des exigences linguistiques qu'à d'autre reste donc ouverte.

Comparativement au domaine du marché intérieur, où les questions de traduction sont toujours traitées selon leur incidence sur les libertés de circulation, dans le domaine émergent de la coopération judiciaire civile, ces questions sont traitées pour elles-mêmes, principalement en raison de leur lien étroit avec les droits de la défense. 


\section{Coopération judiciaire en matière civile}

Au cours des dernières années, le droit communautaire a investi un champ jusque là réservé au droit international privé, celui de la coopération judiciaire en matière civile. Les instruments adoptés dans ce domaine traitent de plusieurs manières de la traduction (traduction d'actes ou de pièces dans des procédures transfrontalières). De manière générale, ils reflètent un souci constant d'économiser des traductions, car celles-ci sont sources à la fois de coûts et de délais. Or, l'un des défis de la construction d'un espace judiciaire européen dans lequel, idéalement, les procédures transfrontalières ne seraient pas plus difficiles à mettre en œuvre que des procédures nationales, tient précisément à la minimisation des coûts et des délais spécifiques à ces procédures dans un contexte multilingue. On assiste donc à travers les règlements communautaires adoptés dans ce domaine à un effort de rationalisation des traductions, spécialement grâce à la technique des formulaires (A). Pour autant, ces règlements créent également des obligations de traduction, spécialement lorsque l'exercice des droits de la défense est en cause (B).

\section{A. La traduction rationnalisée par les formulaires}

Pour rationnaliser les coûts de traduction, une technique efficace et abondamment utilisée dans les règlements qui forment le cadre juridique de l'espace judiciaire européen en matière civile consiste dans l'utilisation de formulaires. Tous les règlements en prévoient pour pratiquement toutes les étapes des procédures qu'ils instituent. Ainsi, pour prendre l'exemple du règlement relatif à la procédure européenne de règlement des petits litiges ${ }^{88}$, cette procédure très simplifiée se déroule entièrement par échange de formulaires: le premier pour la demande, le second pour une éventuelle demande d'informations complémentaires émanant de la juridiction saisie, le troisième pour la

88 Règlement (CE) n 861/2007 du Parlement européen et du Conseil du 11 juillet 2007 instituant une procédure européenne de règlement des petits litiges, $J O \mathrm{~L}$ 199 , p. 1-22. 
réponse du défendeur et le dernier pour la décision elle-même ${ }^{89}$. D'autres règlements contiennent en annexe jusqu'à une dizaine de formulaires différents pour couvrir tous les événements procéduraux possibles ${ }^{90}$. Le grand avantage des formulaires est naturellement qu'ils permettent d'automatiser une grande partie de la traduction, et ce d'autant plus facilement à mesure qu'ils seront remplis et transmis via une plateforme électronique adaptée. Ainsi, la juridiction, ou les parties selon le cas, pourront remplir un formulaire dans leur langue et la juridiction, ou l'autorité destinataire pourra visualiser le formulaire dans sa propre langue. La standardisation du formulaire limite le besoin de traduction au contenu spécifique à chaque demande, à l'exclusion de tous les éléments récurrents (rubriques du formulaire). En outre, certaines informations renseignées sur les formulaires n'auront pas besoin d'être traduites (par exemple nom et adresse du destinataire d'un acte à notifier).

La traduction n'est cependant pas entièrement évitable ni automatisable. Ainsi, une juridiction qui formule une demande d'assistance pour le recueil de preuves situées dans un autre État membre devra transmettre à la juridiction requise le formulaire ad hoc traduit dans la langue de cette dernière ou dans une autre langue acceptée par l'État membre requis ${ }^{91}$. D' un règlement à l'autre, en fonction notamment de la complexité des informations échangées, certains États membres acceptent ou non que des formulaires soient transmis à leurs juridic-

89 Le formulaire de demande (formulaire A) à remplir par le demandeur et à adresser à la juridiction compétente. Dans le cas où ce formulaire ne comporterait pas toutes les informations requises, celle-ci peut les demander, en utilisant le formulaire $\mathrm{B}$, prévu à cet effet. Une fois que la demande est complète, la juridiction en accuse réception en complétant une partie du formulaire de réponse (formulaire $\mathrm{C}$, partie I), lequel est alors notifié au défendeur accompagné du formulaire de demande. Le défendeur remplit alors l'autre partie du formulaire C. Un formulaire D est également prévu pour la décision elle-même.

90 Règlement (CE) n 1206/2001 du Conseil du 28 mai 2001 relatif à la coopération entre les juridictions des États membres dans le domaine de l'obtention des preuves en matière civile ou commerciale, $J O$ L 174, p. 1, dont l'annexe contient dix formulaires types.

91 Règlement (CE) n 1206/2001, cité supra note 90, article 5. 
tions ou autorités compétentes dans une langue autre que leur(s) langue(s) officielle(s) ${ }^{92}$.

Au-delà des formulaires, qui visent à limiter les traductions et à en limiter le coût, plusieurs des instruments adoptés dans le domaine de la coopération judiciaire en matière civile traitent de question de traduction proprement dite, car celles-ci peuvent être essentielles à la protection des droits de la défense.

\section{B. Les traductions requises pour garantir les droits de la défense}

Il n'est pas concevable que, dans l'espace judiciaire européen, un défendeur se voit valablement notifier dans une langue qu'il ne comprend pas une assignation devant une juridiction d'un État membre autre que celui où il réside. Aussi, il existe des dispositions réglementaires relatives à la traduction, et ce dans la plupart des règlements. Celles-ci sont cependant rapidement apparues insuffisamment précises et ont d'ores et déjà donné lieu à plusieurs arrêts de la Cour, comme l'illustrent les quelques exemples suivants.

Le règlement sur la signification et la notification des actes judiciaires ${ }^{93}$, applicable aux notifications transfrontalières d'actes judiciaires et extra-judiciaires prévoit que ceux-ci doivent être traduits afin d'être compris par leur destinataire. Plus précisément, le règlement

92 A titre d'exemple, la Belgique n'accepte pas d'autre langue que la langue de la juridiction compétente (français, néerlandais ou allemand selon le cas) dans le cadre du règlement $(\mathrm{CE}) \mathrm{n}^{\circ}$ 1206/2001sur l'obtention des preuves. En revanche, dans le cadre du règlement $(\mathrm{CE}) \mathrm{n}^{\mathrm{o}} 1348 / 2000$ relatif à la signification et à la notification des actes judiciaires et extrajudiciaires (cité infra note 93), sont acceptés en Belgique les formulaires rédigés non seulement dans l'une des trois langues nationales mais également en anglais. Les langues acceptées font l'objet de déclarations des États membres en application de chaque règlement. L'ensemble des informations résultant de ces déclaration est accessible sur un site internet spécialisé, l'Atlas judiciaire européen en matière civile: http://ec.europa.eu/justice_home/ judicialatlascivil/

93 Règlement $(\mathrm{CE}) \mathrm{n}^{\circ}$ 1348/2000 du Conseil du 29 mai 2000 relatif à la signification et à la notification dans les États membres des actes judiciaires et extrajudiciaires en matière civile et commerciale, $J O$ L 160, p. 37. 
impose une traduction soit dans une langue comprise du destinataire soit dans la langue officielle de l'État membre où a lieu la notification $^{94}$. Lors de la notification, le destinataire doit être informé par l'autorité en charge de procéder à la notification qu'il a le droit de refuser de recevoir l'acte si celui-ci n'est pas rédigé ou accompagné d'une traduction dans l'une des langues satisfaisant à ces conditions. De son côté, le requérant doit être avisé de ce droit de refus par l'entité à laquelle il remet l'acte aux fins de transmission ${ }^{95}$. Les frais de traduction qu'implique le respect de ces exigences sont à sa charge $^{96}$.

Ces dispositions relatives à l'exigence de traduction, dans certaines conditions, d'un acte à notifier, et à l'allocation des coûts de traduction présentent plusieurs lacunes. Tout d'abord, le texte du règlement ne précise pas si l'expression «une langue comprise du destinataire» doit s'entendre également d'une langue qui serait certes comprise du destinataire mais ne serait pas une langue officielle de l'Union. A la lettre, le texte de l'article 8 du règlement paraît l'autoriser. Toutefois, un considérant du règlement, dans sa version initiale, tend à montrer que l'intention du législateur communautaire était de permettre aux parties d'économiser des coûts de traduction en autorisant la signification ou la notification dans la langue ou l'une des langues de l'État d'origine, lorsque celle-ci est comprise par le destinataire. En effet, il était précisé dans les considérants liminaires du règlement que «[a]fin

94 Règlement (CE) n 1393/2007 du Parlement européen et du Conseil du 13 novembre 2007 relatif à la signification et à la notification dans les États membres des actes judiciaires et extrajudiciaires en matière civile ou commerciale et abrogeant le règlement $(\mathrm{CE}) \mathrm{n}^{\circ}$ 1348/2000 du Conseil, JO L 324 du 10 décembre 2007 , p. $79-120$, article 8 , paragraphe 1 . Dans le règlement initial (règlement $(\mathrm{CE})$ $\mathrm{n}^{\circ} 1348 / 2000$, article 8 , paragraphe 1 , l'ordre des options était inverse (langue officielle puis langue comprise par le destinataire). Le texte précise que, s'il existe plusieurs langues officielles dans cet État membre, cette seconde possibilité s'entend d'une notification dans la langue officielle ou l'une des langues officielles du lieu où il doit être procédé à la signification ou à la notification.

95 Règlement (CE) nº 1348/2000 cité supra note 93, article 5, paragraphe 1.

96 Règlement (CE) n ${ }^{\circ} 1348 / 2000$ cité supra note 93, article 5, paragraphe 2. Cette règle s'entend, comme le précise le texte, sans préjudice, dans le cas d'une procédure judiciaire, du pouvoir de la juridiction saisie de statuer sur l'allocation des frais et dépens. 
de défendre les intérêts du destinataire, il convient que la signification ou la notification se fasse dans la langue ou l'une des langues officielles du lieu où elle sera effectuée ou dans une autre langue de l'État membre d'origine que le destinataire comprend ${ }^{97} »$. Cette interprétation se retrouve dans un arrêt de la Cour, où la formule de l'article 8 du règlement, «une langue comprise par le destinataire», est remplacée par «une langue de l'État membre d'origine que [le] destinataire comprend ${ }^{98} \gg$. Toutefois, le contexte ne permet pas d'en tirer avec certitude un enseignement général ${ }^{99}$. En outre, ce considérant a disparu du règlement révisé, si bien que l'on peut s'interroger sur la régularité de la notification dans un État membre de l'Union d'un document rédigé ou traduit dans une langue autre qu'une des langues officielles d'un État membre (lorsque le destinataire comprend cette langue). En l'absence de jurisprudence plus précise, et dans le silence du règlement sur ce point, la question des langues admissibles pour la traduction d'un acte judiciaire ou extra-judiciaire relève des droits nationaux, lesquels y apportent sans doute des réponses divergentes et pas nécessairement uniformes selon qu'est en cause un acte judiciaire ou un acte extra-judiciaire ${ }^{100}$.

Deux autres questions d'interprétation des dispositions relative à la traduction dans le règlement sur la notification des actes judiciaires et extra-judiciaires ont en revanche été tranchées par la Cour. Il s'agit, d'une part, de la question de la sanction de l'absence de traduction et,

97 Règlement $(\mathrm{CE}) \mathrm{n}^{\circ}$ 1348/2000 cité supra note 93, considérant 10. Compar. considérant 12 du règlement $(\mathrm{CE}) \mathrm{n}^{\circ} 1393 / 2007$ (cité supra note 94).

98 CJCE, 8 novembre 2005, Leffler, C-443/03, Rec., p. I-9611, point 31.

99 Dans ce point précité, la Cour reformule la question préjudicielle de la Cour de renvoi. L'expression est certes reprise au point 53 de l'arrêt, dans la réponse de la Cour, mais il reste délicat d'en tirer une conclusion générale, car la question de l'emploi d'une langue autre qu'une langue officielle d'un État membre ne se trouvait pas posée en l'espèce.

100 La Cour a récemment eu l'occasion de préciser que la signification d'actes notariés en dehors d'une procédure judiciaire relève du règlement $n^{\circ}$ 1348/2000 (désormais règlement (CE) n 1393/2007). CJCE, 25 juin 2009, Roda Golf \& Beach Resort, C-14/08, non encore publié au Recueil. 
d'autre part, de l'étendue de l'obligation de traduction qui s'impose au requérant.

L'absence de précision du règlement quant à la sanction de l'absence de traduction était au cœur de l'affaire ayant donné lieu à l'arrêt Leffler ${ }^{101}$. Dans cette affaire, le litige au principal opposait un demandeur établi aux Pays-Bas à une société établie en Allemagne. Cette dernière avait été assignée à comparaître devant une juridiction néerlandaise, mais avait refusé de recevoir l'assignation au motif que celle-ci n'était pas accompagnée d'une traduction en allemand. La question qui s'était alors posée au juge néerlandais était de savoir si l'assignation était nulle ou bien si le demandeur pouvait, moyennant l'envoi ultérieur d'une traduction, conserver le bénéfice procédural de l'assignation à laquelle il avait fait procéder. Sur ce point, la Cour juge que le règlement «doit être interprété en ce sens que, lorsque le destinataire d'un acte a refusé celui-ci au motif que cet acte n'est pas rédigé dans une langue officielle de l'État membre requis ou dans une langue de l'État membre d'origine que ce destinataire comprend, l'expéditeur a la possibilité d'y remédier en envoyant la traduction demandée ». La solution peut se justifier au regard de l'adage «pas de nullité sans texte», mais aussi au regard de considérations pragmatiques. Admettre que l'absence de traduction est sanctionnée par la nullité de l'assignation aurait conduit à ce que, en cas de doute, les demandeurs ne prennent jamais le risque de ne pas traduire. Or, comme on l'a vu, l'esprit du règlement sur la notification des actes judiciaires et extra-judiciaires, et plus généralement de la coopération judiciaire, est précisément de réduire les formalités et les coûts propres aux procédures transfrontalières. Dès lors, l'exigence systématique d'une traduction à peine de nullité aurait semblée contraire à l'esprit même de l'espace judiciaire européen en matière civile. En somme, en autorisant la régularisation de l'absence de traduction, la Cour contribue à cantonner celle-ci à son domaine utile. Ce principe est confirmé par l'arrêt Weiss ${ }^{102}$, dans lequel était plus précisément en cause l'étendue de l'obligation de traduction.

${ }^{101}$ CJCE, 8 novembre 2005, Leffler, C-443/03, Rec., p. I-9611.

102 CJCE, 8 mai 2008, Weiss, C-14/07, Rec., p. I-3367. 
L'affaire ayant donné lieu à cet arrêt opposait au principal la chambre de commerce de Berlin à un cabinet d'architectes établi au Royaume-Uni. Celui-ce se voyait réclamer des dommages et intérêts en réparation d'un défaut de conception. Les prestations de conception litigieuses avaient été prestées en exécution d'un contrat d'architecte rédigé en allemand et aux termes duquel il était convenu que la langue de communication entre les parties serait l'allemand ${ }^{103}$. Dans ces circonstances, le demandeur allemand avait pu croire qu'il n'était pas nécessaire de traduire en anglais sa requête avant de la faire signifier au défendeur au Royaume-Uni. Pourtant, lors de la première tentative, la signification de la requête avait été refusée au motif qu'elle n'était pas accompagnée d'une traduction en anglais. La requête avait alors été traduite puis à nouveau signifiée. Là encore, le cabinet d'architecte avait refusé de recevoir le document au motif que, si la requête ellemême était traduite, tel n'était pas le cas de ses annexes, volumineuses de 150 pages et contenant les preuves sur lesquelles s'appuyait le demandeur, avec des références au contrat rédigé en allemand et à des considérations techniques. Ce nouveau refus soulevait la question de l'étendue de l'obligation de traduction. Interrogée sur ce point, la Cour répond que le règlement «doit être interprété en ce sens que le destinataire d'un acte introductif d'instance à notifier ou à signifier n'a pas le droit de refuser la réception de cet acte pour autant que celui-ci met ce destinataire en mesure de faire valoir ses droits dans le cadre d'une procédure judiciaire dans l'État membre d'origine ${ }^{104} »$. Elle précise que ne sont pas soumises à l'obligation de traduction les annexes «constituées de pièces justificatives [...] qui ont uniquement une fonction de preuve et ne sont pas indispensables pour comprendre l'objet et la cause de la demande». Le juge national est donc invité à vérifier au cas par cas «si le contenu de l'acte introductif d'instance est suffisant pour permettre au défendeur de faire valoir ses droits ou s'il incombe à l'expéditeur de remédier à l'absence de traduction d'une annexe indispensable». C'est donc le respect des droits de la défense

103 Arrêt Weiss, cité supra note 102, points 19 et 20.

104 Arrêt Weiss, cité supra, point 78. 
qui constitue le principe limitatif permettant de fixer l'étendue de l'obligation de traduction pesant sur le demandeur.

Il semble donc que la Cour interprète les exigences de traduction tant quant à la sanction de leur non-respect que quant à la détermination de leur étendue - au regard d'un principe de proportionnalité. En effet, dans un cas, la sanction de la nullité serait disproportionnée et dans l'autre la traduction requise est celle qui est nécessaire et proportionné à l'exercice des droits de la défense. Ce constat rejoint celui d'un autre auteur, pour qui la proportionnalité est le critère décisif qui se dégage de la jurisprudence de la Cour en matière d'exigences linguistiques ${ }^{105}$.

\section{Conclusion}

Ce survol des questions de traduction telles qu'elles apparaissent en droit communautaire montre leur grande diversité : la traduction n'est pas appréhendée de manière unitaire ou cohérente. Elle est parfois l'objet de règles spécifiques (comme dans le domaine de la coopération judiciaire en matière civile) mais tombe également sous le coup de règles qui ont un tout autre objet (par exemple le droit des marques). Aussi, n'y a-t-il pas de régime général de la traduction en droit communautaire.

Dans le droit institutionnel, les langues ne sont égales que dans la mesure prévue par les traités et par le règlement $\mathrm{n}^{\circ} 1$. Pour le surplus, le refus de la Cour de reconnaître un principe général d'égalité des langues permet une certaine rationalisation du multilinguisme des institutions et organes de l'Union et, partant, une limitation des exigences de traduction dans toutes les langues officielles.

Dans le droit du marché intérieur, les exigences nationales ou privées relatives à l'emploi des langues et aux traductions sont appréhendées à travers le prisme de leur effet sur les libertés de circulation. Si certaines exigences nationales relatives à l'emploi d'une langue peuvent être contraires au droit dérivé ou qualifiées d'entraves au bon

105 J. VAnHAmme, «L'équivalence des langues dans le marché intérieur : l'apport de la Cour de justice», CDE, 2007, p. 359-380, spéc. p. 376 et s. 
TRADUCTION ET DROITS EUROPÉENS

fonctionnement du marché intérieur, ce n'est pas le cas général. La jurisprudence de la Cour démontre au contraire que les exigences de traduction des États membres peuvent être justifiées par des exigences impératives d'intérêt général.

Enfin, dans le domaine de la coopération judiciaire en matière civile, la traduction apparaît à la fois comme une source de délais et comme une nécessité. Les instruments existants s'efforcent de rationaliser ces coûts et délais mais prévoient également des obligations de traduction, notamment là où elles paraissent nécessaires à l'exercice des droits de la défense. Dans l'interprétation qu'elle a donné de ces textes, la Cour semble avoir été guidée par le principe de proportionnalité. Finalement, c'est peut être ce principe, si central en droit communautaire, qui permet de relier les règles rencontrées dans les différentes branches du droit communautaire. Des traductions sont exigées (par le droit communautaire lui-même, par les droits nationaux, par les particuliers); ces exigences ne sont que partiellement encadrées par des textes, si bien que leur légalité reste largement soumise à l'examen de leurs justifications et de leur proportionnalité. 